Research Paper

\title{
Clinical characteristics and prognostic analysis of Lung Cancer patients with Hypercoagulability: A single-center, retrospective, real-world study
}

\author{
Yunfei Ma${ }^{1}$, Guangda $\mathrm{Li}^{*}$, Xiaoxiao Li ${ }^{1 *}$, Yu Gao², Tongjing Ding ${ }^{2}$, Guowang Yang1, Yi Zhang1, Jiayun \\ Nian ${ }^{1}$, Mingwei $\mathrm{Yu}^{1 凶}$ and Xiaomin Wang ${ }^{\circledR}$ \\ 1. Beijing Hospital of Traditional Chinese Medicine, Capital Medical University, Beijing, 100010, China. \\ 2. Beijing University of Chinese Medicine, Beijing, 100029, China. \\ ${ }^{*}$ Co-second authors with equal contributions to this work. \\ $\triangle$ Corresponding authors: Dr. Xiaomin Wang, E-mail: wangxiaomin_bhtcm@126.com; ORCID: https://orcid.org/0000-0003-1274-224X. Dr. Mingwei Yu, \\ E-mail: yumingwei@ccmu.edu.cn; ORCID: https://orcid.org/0000-0001-9224-4085.
}

(c) The author(s). This is an open access article distributed under the terms of the Creative Commons Attribution License (https://creativecommons.org/licenses/by/4.0/). See http://ivyspring.com/terms for full terms and conditions.

Received: 2020.04.02; Accepted: 2021.03.01; Published: 2021.03.19

\begin{abstract}
Objective: We explored the clinical regularity and prognosis of lung carcinoma (LC) patients with hypercoagulability, which is often associated with the occurrence and development of tumors.

Methods: This retrospective study analyzed 624 LC patients diagnosed from 2010-2017 in the Beijing Hospital of Traditional Chinese Medicine, Capital Medical University, China. Kaplan-Meier analysis was used to estimate survival and the log-rank test was used to identify differences in survival between groups. The predictive power of a hypercoagulation model was tested using receiver operating characteristic (ROC) curve analysis. Univariate and multivariate Cox regression analyses were performed to explore independent factors associated with survival. A logistic regression model was used to explore factors related to hypercoagulability. The diagnostic power of relevant influencing factors on hypercoagulability was tested using ROC curve analysis.

Results: Of 624 patients in the study, 161(25.8\%) had hypercoagulability and $463 \mathrm{did}$ not (normal group). The overall survival (OS) of the hypercoagulability group was significantly lower than the normal group $(P<0.0001)$. The ROC curve showed that the predictive power of the hypercoagulability model was better than that of a single coagulation indicator $(P<0.01)$. Both univariate and multivariate Cox regression analyses showed that hypercoagulability was an independent factor affecting the prognosis of LC $(P<0.0001)$. The results of the logistic regression analysis showed that clinical stage $(P<0.05)$, cytokeratin 19 fragment (Cyfra211) $(P<0.05)$, and the platelet-to-lymphocyte ratio $(P L R)(P<0.05)$ were positively correlated with hypercoagulability. When combining clinical stage, Cyfra211, and the PLR to predict hypercoagulability, the area under the ROC curve was $0.797(P<0.01)$

Conclusions: In LC, hypercoagulability is an independent factor associated with poor OS and could be a prognostic factor.
\end{abstract}

Key words: lung neoplasms; hypercoagulability; clinical characteristics; prognosis; real-world study

\section{Introduction}

Lung carcinoma (LC) is the leading cause of cancer-related death. Of the 9.56 million people who died of cancer in 2018, 1.76 million (18.4\%) had LC [1]. With the advance of targeted therapy and immunotherapy, great progress has been made in the treatment of LC, but the 5-year survival rate of LC is still dismal $[2,3]$. Therefore, the ability to identify effective prognostic factors for predicting the clinical outcomes of LC is critical to making treatment decisions.

Hypercoagulability is a state of vascular endothelial cell injury, decreased anticoagulation function, and decreased tissue fibrinolytic activity caused by a variety of factors, which lead to increased coagulation of blood. Hypercoagulability in cancer patients is closely associated with the activation of the 
coagulation system and an increased number of platelets (PLTs). Some studies have demonstrated that hypercoagulability can affect the biological behavior of tumor cells, including promoting proliferation, invasion, and immune escape $[4,5]$. The relationship between coagulation functions and malignancy has been extensively studied [6-8], revealing an association between the prognoses of LC patients and single coagulation-related factors, including levels of PLTs, D-dimer, and fibrinogen (FIB). However, the results of these studies are inconsistent. A multicenter prospective study showed a significant association between an elevated PLT count and poor progression-free survival (PFS), whereas levels of FIB and D-dimer were not significant prognostic factors [9]. Hong et al. [10] and $\mathrm{Wu}$ et al. [11] suggested that an elevated PLT count was not a prognostic factor of LC. Jiang et al. [12] reported that elevated D-dimer significantly predicted poor survival of LC.

In order to comprehensively evaluate the prognosis of patients with LC, we combined multiple coagulation indicators and constructed a hypercoagulation model based on the study of Yang et al. [13] to evaluate the prognostic value of hypercoagulability and explore the clinical characteristics of LC patients with hypercoagulability. The goal of our study was to provide a new perspective for better understanding of the association between hypercoagulability and prognosis in LC.

\section{Methods}

\section{Study design}

This was a single-center, retrospective, real-world study. The Information Center of the Beijing Hospital of Traditional Chinese Medicine, affiliated to the Capital Medical University (BHTCM) assisted in collecting the information of LC patients in the Hospital Information System (HIS) database. All data were from the first-admission evaluations in our hospital. In the BHTCM HIS system, patients with LC who visited the hospital from January 2010 to December 2017 were screened through the first page of medical records. The search terms included the following: "neoplasms," "lung," "lung neoplasm," "carcinoma," "non-small-cell lung," "carcinoma, bronchogenic," "adenocarcinoma of lung," "carcinoma, squamous cell," "small cell lung carcinoma", "carcinoma, large cell," and "pulmonary nodule." Dates of patient death were obtained from the Beijing Centers for Disease Control and Prevention, China.

\section{Participants}

All patients in this study were admitted to the
BHTCM from January 2010 to December 2017. The inclusion criteria were a pathological diagnosis of LC, age $\geq 18$, and at least two indicators of coagulation function. The exclusion criteria were other tumors, coronary heart disease, cerebral infarction, hemopathy, acute inflammatory reaction, non-tumor-related surgery within the last month, and administration of drugs affecting blood coagulation or anticoagulant function within the last month. Hypercoagulability was defined as meeting at least two of the following criteria: elevated FIB levels, shortened prothrombin time, shortened activated partial thromboplastin time, elevated D-dimer levels, and increased PLTs [13]. This study was approved by the institutional review board (2017 BL-087-03).

\section{Data collection}

Clinically relevant data for the first admission of all patients, including general patient information, pathological characteristics, and relevant examinations, were collected by oncology professionals.

\section{Statistical analysis}

Data were exported from EpiData version 3.1 (EpiData Association, Odense, Denmark) to SPSS Version 22.0 (IBM, Armonk, NY, USA) for analysis. Descriptive statistics were performed for patient characteristics, pathological features, and relevant examinations. Quantitative variables were expressed as the mean \pm standard deviation, and comparisons were performed by the Mann-Whitney $U$ test. Categorical variables were expressed as N (\%), and the chi-square test was used for comparison. Kaplan-Meier analysis was used to estimate survival, and the log-rank test was used to identify differences in survival between groups. The predictive power of a model was tested using receiver operating characteristic (ROC) curve analysis. Univariate and multivariate Cox regression analyses were performed to explore independent factors associated with survival. A logistic regression model was used to explore factors related to hypercoagulability. The diagnostic power of relevant influencing factors on hypercoagulability was tested using ROC curve analysis. A $P$-value $<0.05$ was considered statistically significant.

\section{Results}

\section{Patient characteristics}

The initial search retrieved 10,502 records. After meticulous inspection of the patient information, 624 inpatients from 2010-2017 were enrolled in this study. The patient selection process was shown in Figure 1 and the characteristics of the included patients were 
shown in Table 1. There were no significant differences in gender, process, smoking status, alcohol consumption, tumor location, or pathological type between LC patients with hypercoagulability (hypercoagulability group) and those without (normal group) ( $P>0.05$ for each). There were statistically significant differences in age, body mass index (BMI), clinical stage, carcinoembryonic antigen level (CEA), neuron-specific enolase level (NSE), cytokeratin 19 fragment level (Cyfra211), platelet-tolymphocyte ratio (PLR), and neutrophil-tolymphocyte ratio $(\mathrm{NLR})$ between the groups $(P<0.05$ for each).

\section{Kaplan-Meier analysis for overall survival}

As shown in Figure 2, the hypercoagulability group (HR: 2.128, 95\% CI: 1.664-2.721), the high-D-dimer group (HR: 1.922, 95\% CI: 1.325-2.786), the high-FIB group (HR: 2.074, 95\% CI: 1.697-2.534), and the high-PLT group (HR: 1.851, 95\% CI: 1.515-2.263) had significantly lower overall survival (OS) than the normal group $(P<0.0001$ for each). The median survival times of the hypercoagulability and normal groups were 5.5 months and 14.1 months, respectively.

\section{ROC curves of PLT, D-dimer, FIB and hypercoagulability}

The area under the curve (AUC)

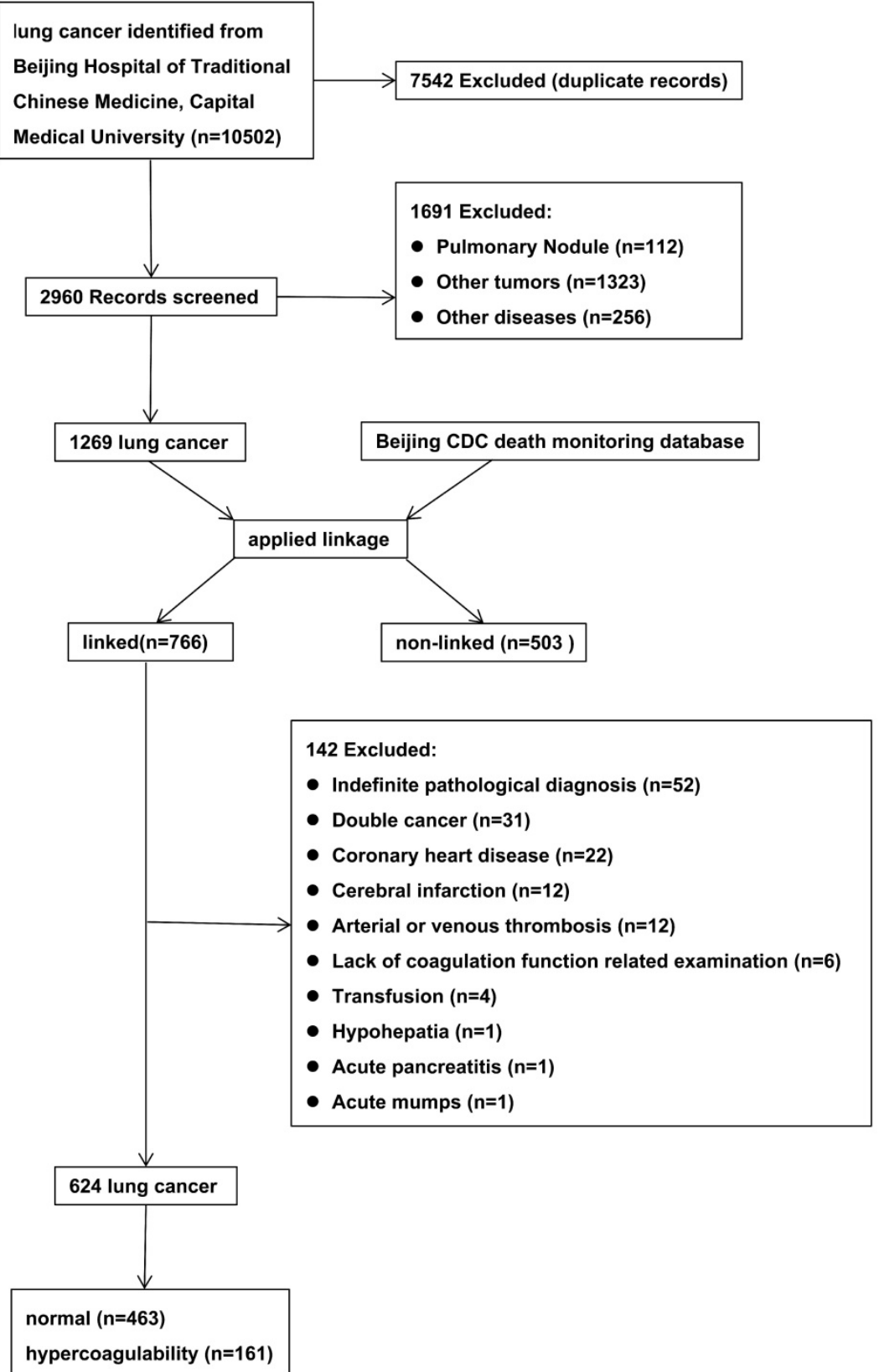
reflects predictive accuracy [14]. As shown in Figure 3, the AUCs of PLT, D-dimer, FIB and hypercoagulability were 0.571 (95\% CI: $0.525-0.617), 0.659$ (95\% CI: $0.615-0.704), \quad 0.652(95 \%$ CI: $0.607-0.696)$, and $0.686 \quad(95 \% \quad \mathrm{CI}$ : $0.646-0.727)$, respectively $(P<0.01$ for each).

\section{Univariate analysis for overall survival}

Based on the analysis of the patient characteristics, we further analyzed the relationships between prognosis and age, stage, CEA, NSE, Cyfra211, NLR, PLR, and hypercoagulability. Univariate analysis revealed that hypercoagulability was significantly associated with decreased OS (HR: 2.154, 95\% CI: 1.755-2.645, $P=0.000)$. Additionally, age, stage, NSE, Cyfra211, NLR, and PLR were significantly associated with poor OS in LC (Figure 4).

\section{Multivariate analysis of overall survival}

As summarized in Table 2, a multivariate Cox regression analysis was performed using hypercoagulability, age, stage, PLR, NLR, CEA, NSE, and Cyfra211 to investigate the independent prognostic factors for OS of LC. Hypercoagulability was still an independent prognostic factor associated with poor OS (HR: 1.591, $95 \%$ CI: 1.262-2.005, $P=0.000)$, along with stage, PLR and NSE. 


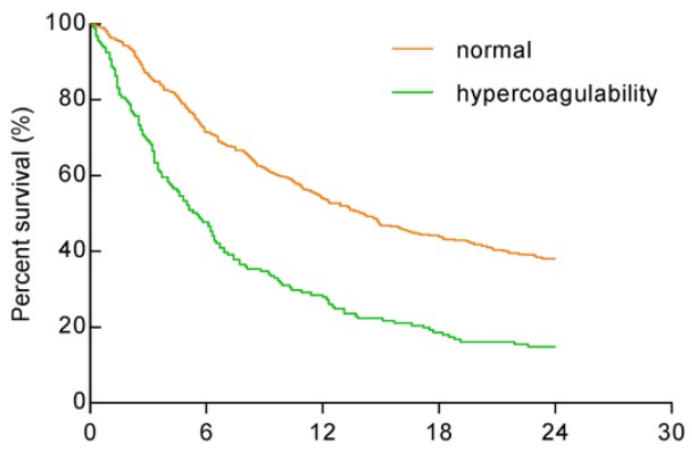

C

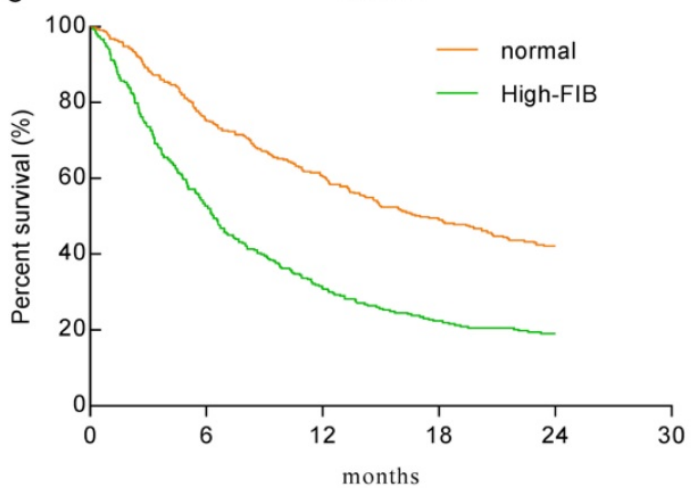

B

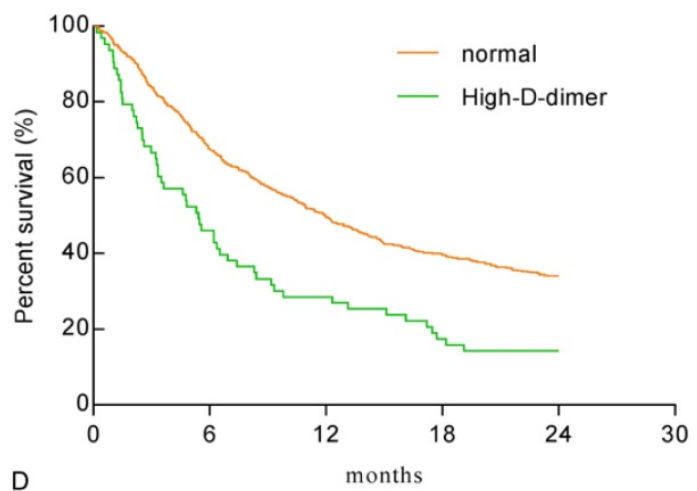

D

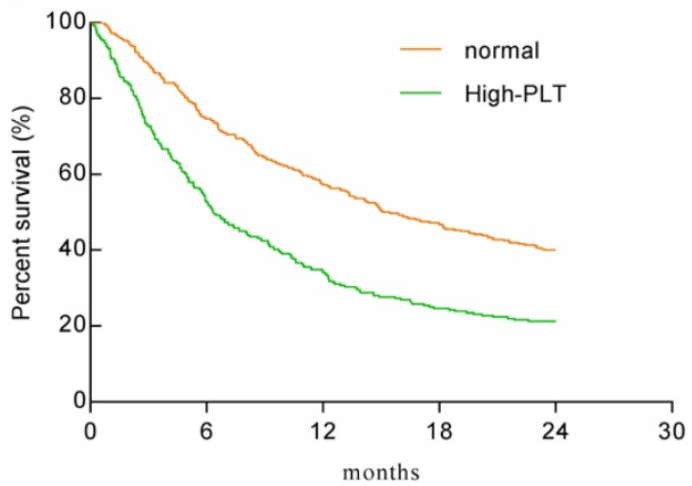

Figure 2. Kaplan-Meier plots for the total population. A. The hypercoagulability group VS the normal group (HR: $2.128,95 \% \mathrm{Cl}: 1.664-2.721$; $P<0.0001)$. B. The high-D-dimer group VS the normal group (HR: 1.922, 95\% Cl: 1.325-2.786; $P<0.0001)$. C. The high-FIB group VS the normal group $(\mathrm{HR}: 2.074,95 \% \mathrm{Cl}$ : $1.697-2.534 ; P<0.0001)$. D. The high-PLT group VS the normal group (HR: 1.851, 95\% Cl: 1.515-2.263; $P<0.0001)$.

Table 1. Main characteristics of all the patients included in the study

\begin{tabular}{|c|c|c|c|c|}
\hline Characteristics of cases & Hypercoagulability & Normal & $x^{2} / Z$ & $P$ \\
\hline Age (years) & & & 6.825 & 0.033 \\
\hline $18-45$ & $8(5 \%)$ & $9(1.91 \%)$ & & \\
\hline $46-65$ & $60(37.3 \%)$ & $213(46.0 \%)$ & & \\
\hline $66-85$ & $93(57.8 \%)$ & $241(52.1 \%)$ & & \\
\hline Process (years) & & & 4.736 & 0.192 \\
\hline$<1$ & $116(72.0 \%)$ & $297(64.1 \%)$ & & \\
\hline$\geq 1-<2$ & $19(11.8 \%)$ & $69(14.9 \%)$ & & \\
\hline$\geq 2-<5$ & $21(13.0 \%)$ & $66(14.3 \%)$ & & \\
\hline$\geq 5$ & $5(3.1 \%)$ & $31(6.7 \%)$ & & \\
\hline \multicolumn{5}{|l|}{ Gender } \\
\hline Man & $95(59.0 \%)$ & $276(59.6 \%)$ & 0.018 & 0.893 \\
\hline Woman & $66(41.0 \%)$ & $187(40.4 \%)$ & & \\
\hline Smoke & & & 0.128 & 0.721 \\
\hline Yes & $84(52.2 \%)$ & $234(50.5 \%)$ & & \\
\hline No & $77(47.8 \%)$ & $229(49.5 \%)$ & & \\
\hline Drink & & & 0.46 & 0.831 \\
\hline Yes & $50(31.1 \%)$ & $148(32.0 \%)$ & & \\
\hline No & $111(68.9 \%)$ & $315(68.0 \%)$ & & \\
\hline Tumor location & & & 0.461 & 0.497 \\
\hline Left lung & $71(44.1 \%)$ & $190(41.0 \%)$ & & \\
\hline Right lung & $90(55.9 \%)$ & $273(59.0 \%)$ & & \\
\hline BMI* & & & 6.981 & 0.031 \\
\hline$<18.5$ & $9(7.5 \%)$ & $20(6.8 \%)$ & & \\
\hline$\geq 18.5-\leq 23.9$ & $80(66.7 \%)$ & $157(53.8 \%)$ & & \\
\hline$\geq 24$ & $31(25.8 \%)$ & $115(39.4 \%)$ & & \\
\hline Pathological type & & & 3.766 & 0.288 \\
\hline adenocarcinoma & $109(67.7 \%)$ & $291(62.9 \%)$ & & \\
\hline squamous carcinoma & $33(20.5 \%)$ & $96(20.7 \%)$ & & \\
\hline small cell lung cancer & $14(8.7 \%)$ & $66(14.3 \%)$ & & \\
\hline other & $5(3.1 \%)$ & $10(2.2 \%)$ & & \\
\hline
\end{tabular}

\begin{tabular}{lllll}
\hline Characteristics of cases & Hypercoagulability Normal & $\chi^{2} / Z$ & $P$ \\
\hline Clinical stage & \multicolumn{5}{c}{35.479} & 0.000 \\
I-III/limited stage & $18(11.2 \%)$ & $167(36.1 \%)$ & & \\
IV/extensive stage & $143(88.8 \%)$ & $296(63.9 \%)$ \\
CEA $(\mathrm{ng} / \mathrm{ml})$ & $114.37 \pm 455.61$ & $59.24 \pm 159.06$ & -3.288 & 0.001 \\
NSE $(\mathrm{ng} / \mathrm{ml})$ & $34.22 \pm 53.28$ & $24.97 \pm 38.46$ & -2.757 & 0.000 \\
Cyfra211 $(\mathrm{ng} / \mathrm{ml})$ & $37.46 \pm 85.01$ & $12.71 \pm 39.24$ & -7.203 & 0.000 \\
PLR & $315.00 \pm 205.06$ & $196.38 \pm 131.91$ & -8.848 & 0.000 \\
NLR & $6.49 \pm 4.79$ & $4.46 \pm 5.15$ & -7.022 & 0.000 \\
\hline *For some reasons, some patients' height or weight data are missing in the HIS \\
system. Therefore, the BMI analysis in this article is only applied to 412 patients.
\end{tabular}

\section{Factors associated with hypercoagulability in LC patients}

As shown in Table 1, there were statistically significant differences in age, BMI, clinical stage, CEA, NSE, Cyfra211, PLR, and NLR between the groups ( $P$ $<0.05$ for each). We conducted logistic regression analysis to examine the correlation between these variables and hypercoagulability. The results showed that clinical stage (odds ratio $[\mathrm{OR}]=3.672, P<0.05$ ), Cyfra211 (OR = 1.007, $P<0.05)$, and the PLR (OR = 1.006, $P<0.05)$ were positively correlated with hypercoagulability (Table 3).

\section{Clinical stage, Cyfra211, and the PLR predict hypercoagulability}

As shown in Figure 5, when using clinical stage, Cyfra211, and the PLR alone to predict 




Figure 3. The ROC curves of PLT, D-dimer, FIB and the hypercoagulability.

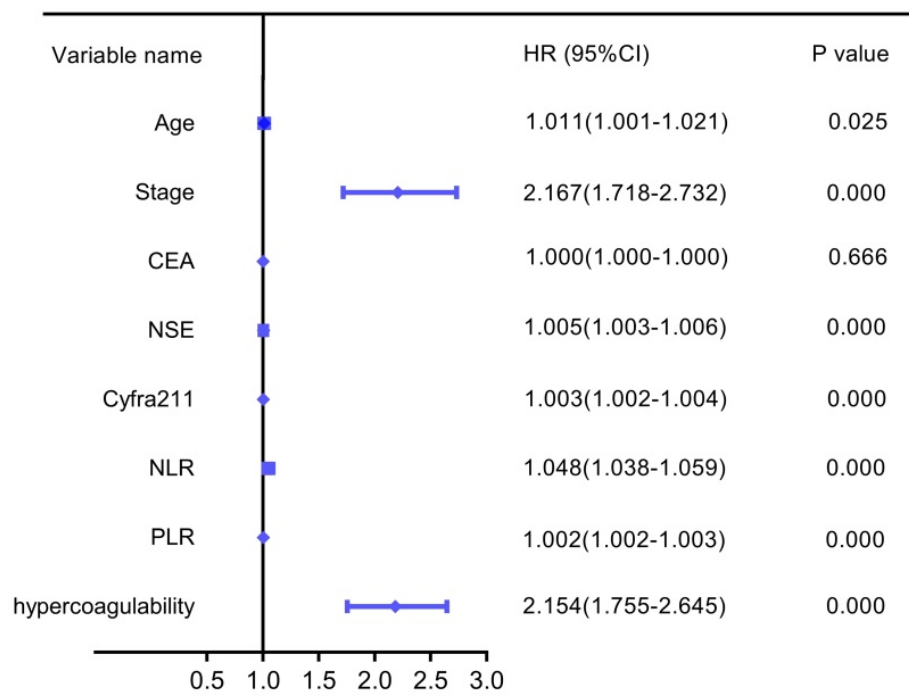

Figure 4. Univariate forest plot for overall survival.

hypercoagulability, the AUCs were 0.622 (95\% CI: 0.564-0.679, $P<0.01), 0.724$ (95\% CI: 0.669-0.779, $P<$ 0.01 ), and 0.750 (95\% CI: 0.695-0.804, $P<0.01$ ), respectively. When using these variables together to predict hypercoagulability, the AUC was 0.797 (95\% CI: 0.749-0.845, $P<0.01$ ).

\section{Discussion}

Metastasis is the leading cause of LC-related death. The pathological mechanism of LC metastasis is extremely complex. Evidence suggests that the coagulation-fibrinolysis system is closely related to the progression of $\mathrm{LC}$ and that activation of coagulation is common in LC $[15,16]$. A previous prospective study demonstrated that LC patients had higher blood coagulation parameters than healthy volunteers, including PLTs, FIB, thrombomodulin, and D-dimer [17]. A retrospective study in Poland found that the frequency of thrombocytosis in surgically treated patients with non-small cell lung cancer (NSCLC) was 10.2\% [18]. Another study of small cell lung cancer found that $64.6 \%$ of patients had high levels of D-dimer [19]. A retrospective study of 856 patients with NSCLC found that $43.6 \%$ of patients had elevated FIB [20]. In this study, 161 patients (25.8\%) had hypercoagulability. Although the mechanism of hypercoagulability in LC has not been elucidated, some associated factors have been reported. Tissue factor is the cell membrane receptor of the serine proteinase coagulation factor VII, whose physiological function is to trigger the activation of the coagulation cascade and increase blood coagulation [21]. It is the most widely studied tumor coagulation factor and is considered to play a central role in the pathophysiology of hypercoagulability [22]. In addition, inflammatory factors play a significant role in coagulation. These factors not only damage endothelial cells and promote angiogenesis, but also increase the expression of cell surface adhesion molecules [23]. Activation of the contact system [24] and thrombocytosis [25] are closely associated with the development of hypercoagulability in cancer.

We demonstrated that hypercoagulability was an independent factor affecting the prognosis of LC and the predictive power of hypercoagulability was better than that of any single coagulation indicator. The hypercoagulability group had significantly lower OS than the normal group. Studies have previously shown that hypercoagulability is a common characteristic of cancer patients and is closely correlated with tumor growth and metastasis [26,27]. In addition, hypercoagulability predisposes patients to venous thromboembolisms, pulmonary embolism, 


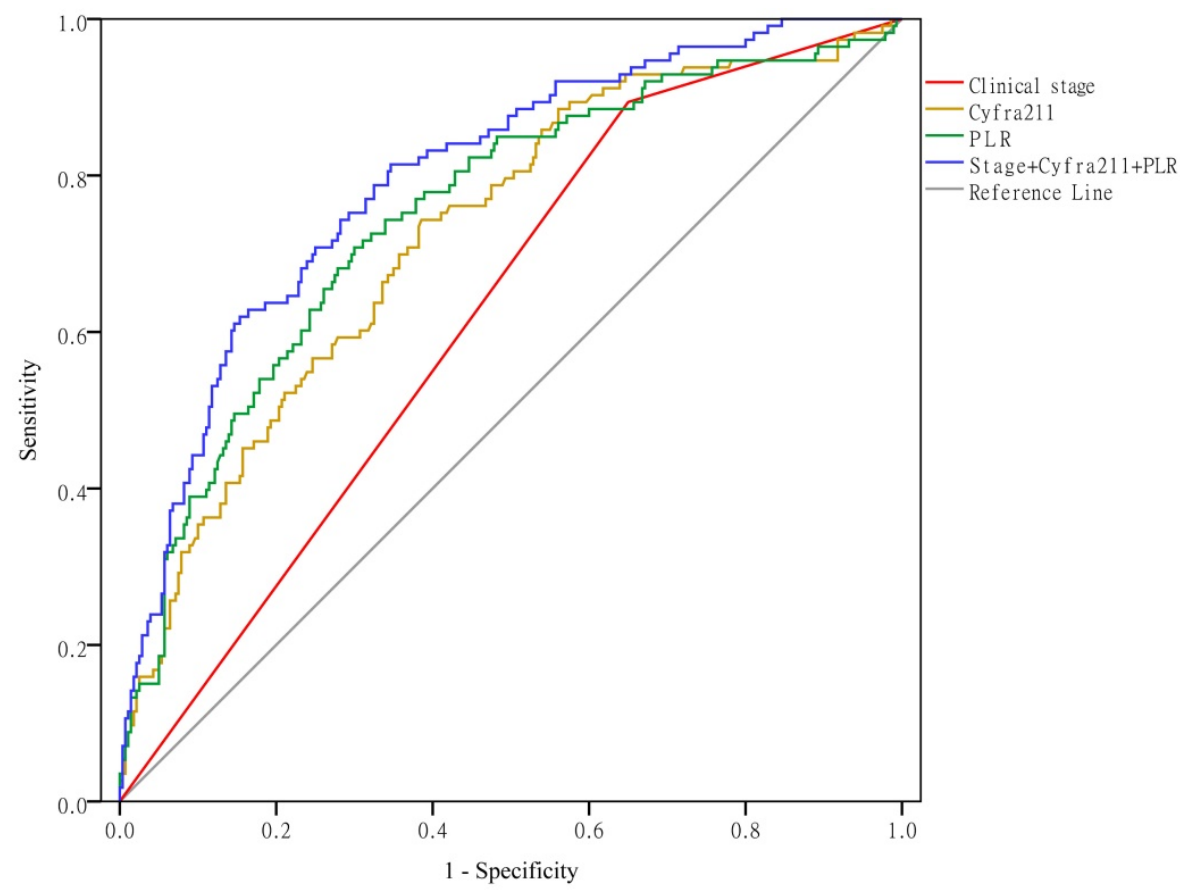

Figure 5. Clinical stage, Cyfra211, and the PLR predict hypercoagulability.

and disseminated intravascular coagulation, which affect the prognosis of LC patients. The result of the logistic regression analysis showed that clinical stage, Cyfra211 and the PLR were positively correlated with hypercoagulability in LC patients. The relationship between clinical stage and thrombocytosis has been observed in previous studies [28,29]. Numerous studies have reported the prognostic significance of the PLR in cancer [30,31].

Table 2. Multivariate Cox regression analysis for overall survival

\begin{tabular}{llllllll}
\hline Covariates & B & S.E. & Wald & Sig. & Exp(B) & \multicolumn{2}{l}{ 95\% CI for EXP(B) } \\
\cline { 6 - 8 } & & & & & & Lower & Upper \\
\hline Age & 0.010 & 0.005 & 3.755 & 0.053 & 1.010 & 1.000 & 1.020 \\
Stage & 0.545 & 0.126 & 18.660 & 0.000 & 1.725 & 1.347 & 2.209 \\
PLR & 0.001 & 0.000 & 6.515 & 0.011 & 1.001 & 1.000 & 1.002 \\
NLR & 0.019 & 0.010 & 3.401 & 0.065 & 1.019 & 0.999 & 1.040 \\
CEA & 0.000 & 0.000 & 1.185 & 0.276 & 1.000 & 1.000 & 1.000 \\
NSE & 0.004 & 0.001 & 18.468 & 0.000 & 1.004 & 1.002 & 1.005 \\
Cyrea211 & 0.001 & 0.001 & 3.641 & 0.056 & 1.001 & 1.000 & 1.003 \\
Hypercoagulability & 0.464 & 0.118 & 15.421 & 0.000 & 1.591 & 1.262 & 2.005 \\
\hline
\end{tabular}

Table 3. Logistic regression analysis of hypercoagulability and Age, BMI, Clinical stage, CEA, NSE, Cyfra211, PLR, NLR

\begin{tabular}{lllllllll}
\hline Dependent & Covariates B & S.E. & Wald & Sig. Exp(B) $\begin{array}{l}\text { 95\% CI for } \\
\text { EXP(B) }\end{array}$ \\
\cline { 6 - 9 } & & & & & & & Lower & Upper \\
\hline Hypercoagulability & Clinical & 1.301 & .364 & 12.761 & .000 & 3.672 & 1.799 & 7.497 \\
& stage & & & & & & & \\
& Cyfra211 & 0.007 & .003 & 6.311 & .012 & 1.007 & 1.002 & 1.012 \\
& PLR & 0.006 & .001 & 32.995 & .000 & 1.006 & 1.004 & 1.007 \\
& Constant & -4.752 & .722 & 43.274 & .000 & .009 & & \\
\hline
\end{tabular}

The aim of this retrospective study was to explore the clinical regularity and prognosis of LC patients with hypercoagulability. The main strength of this study is that it is a realworld study with a relatively large sample size and objective information. Furthermore, this is the first study to apply a hypercoagulation model to analyze the relationship of hypercoagulability and prognosis in patients with LC. This study provides a new perspective for better understanding of the relationship between hypercoagulability and prognosis in LC. Nevertheless, this study has several limitations. The study used a retrospective design, which might induce potential confounding factors. The analysis was limited due to the lack of data on important aspects of treatment, such as surgery, chemotherapy, radiotherapy, targeted therapy, and immunotherapy. Therefore, it is necessary to validate this conclusion in prospective multicenter studies that include stratified analyses according to patient characteristics.

\section{Conclusion}

Hypercoagulability is an independent factor associated with poor OS and could be a prognostic factor for LC.

\section{Abbreviations}

LC: lung carcinoma; PLT: platelet; PFS: progression-free survival; BHTCM: Beijing Hospital of Traditional Chinese Medicine; HIS: Hospital Information System; FIB: fibrinogen; ROC: receiver operative characteristic; BMI: body mass index; HR: hazard ratio; $\mathrm{CI}$ : confidence interval; OS: overall survival; CEA: carcinoembryonic antigen; NSE: neuron-specific enolase; Cyfra211: cytokeratin 19 fragment; OR: odds ratio; PLR: platelet-to-lymphocyte ratio; NLR: neutrophil-to-lymphocyte ratio; AUC: area under the curve; NSCLC: non-small cell lung cancer.

\section{Acknowledgements}

The research was supported through Beijing Municipal Science and Technology Commission (No. Z191100008319006) and Beijing Natural Science Foundation (No. 7202065). 


\section{Author contributions}

Xiaomin Wang, Mingwei $\mathrm{Yu}$ and Yunfei Ma conceived and designed the study. Yunfei $\mathrm{Ma}$, Guangda Li, Xiaoxiao Li, Yu Gao, Tongjing Ding collected the data. Yunfei Ma, Guangda Li, Mingwei $\mathrm{Yu}$ did the statistical analyses. Guowang Yang, Yi Zhang, Jiayun Nian, Mingwei Yu and Xiaomin Wang interpreted the results. Yunfei Ma, Guangda Li, Xiaoxiao Li, Mingwei $\mathrm{Yu}$ and Xiaomin Wang wrote and reviewed the manuscript.

\section{Competing Interests}

The authors have declared that no competing interest exists.

\section{References}

1. Bray F, Ferlay J, Soerjomataram I, Siegel RL, Torre LA, Jemal A. Global cancer statistics 2018: GLOBOCAN estimates of incidence and mortality worldwide for 36 cancers in 185 countries. CA Cancer J Clin. 2018; 68: 394-424.

2. The L. Lung cancer: some progress, but still a lot more to do. Lancet (London, England). 2019: 394: 1880.

3. Han W, Du X, Liu M, Wang J, Sun L, Li Y. Increased expression of long noncoding RNA SNHG16 correlates with tumor progression and poor prognosis in non-small cell lung cancer. Int J Biol Macromol. 2019; 121: 270-8.

4. Krenn-Pilko S, Langsenlehner U, Stojakovic T, Pichler M, Gerger A, Kapp KS, et al. An elevated preoperative plasma fibrinogen level is associated with poor disease-specific and overall survival in breast cancer patients. Breast (Edinburgh, Scotland). 2015; 24: 667-72.

5. Zhang F, Wang Y, Sun P, Wang ZQ, Wang DS, Zhang DS, et al. Fibrinogen promotes malignant biological tumor behavior involving epithelialmesenchymal transition via the p-AKT/p-mTOR pathway in esophageal squamous cell carcinoma. Journal of cancer research and clinical oncology. 2017; 143: 2413-24

6. Qi Y, Fu J. Research on the coagulation function changes in non-small-cell lung cancer patients and analysis of their correlation with metastasis and survival. Journal of BUON: official journal of the Balkan Union of Oncology. 2017; 22: 462-7.

7. Tas F, Kilic L, Serilmez M, Keskin S, Sen F, Duranyildiz D. Clinical and prognostic significance of coagulation assays in lung cancer. Respiratory medicine. 2013; 107: 451-7.

8. Altiay G, Ciftci A, Demir M, Kocak Z, Sut N, Tabakoglu E, et al. High plasma D-dimer level is associated with decreased survival in patients with lung cancer. Clinical oncology (Royal College of Radiologists (Great Britain)). 2007; 19: $494-8$

9. Hou C, Jiang $F$, Ma $H$, Zhu $Q$, Wang $Z$, Zhao B, et al. Prognostic role of preoperative platelet, fibrinogen, and D-dimer levels in patients with non-small cell lung cancer: A multicenter prospective study. Thoracic cancer. 2019; 10: 304-11.

10. Hong X, Xu Q, Yang Z, Wang M, Yang F, Gao Y, et al. The value of prognostic factors in Chinese patients with small cell lung cancer: A retrospective study of 999 patients. The clinical respiratory journal. 2018; 12: 433-47.

11. Wu G, Yao Y, Bai C, Zeng J, Shi D, Gu X, et al. Combination of platelet to lymphocyte ratio and neutrophil to lymphocyte ratio is a useful prognostic factor in advanced non-small cell lung cancer patients. Thoracic cancer. 2015; 6: 275-87.

12. Jiang HG, Li J, Shi SB, Chen P, Ge LP, Jiang $Q$, et al. Value of fibrinogen and $D$-dimer in predicting recurrence and metastasis after radical surgery for non-small cell lung cancer. Medical oncology (Northwood, London, England). 2014; 31: 22.

13. Zheng $Y$, Sun $P$, Dong $Q$, Chen $X Y$. Investigate on the mechanism and diagnostic criteria of hypercoagulable state in malignant tumor. Chinese J Medicine. 2014; 5: 16-20.

14. Babiloni C, Del Percio C, Pascarelli MT, Lizio R, Noce G, Lopez S, et al. Abnormalities of functional cortical source connectivity of resting-state electroencephalographic alpha rhythms are similar in patients with mild cognitive impairment due to Alzheimer's and Lewy body diseases. Neurobiology of aging. 2019; 77: 112-27.

15. Gabazza EC, Taguchi O, Yamakami T, Machishi M, Ibata H, Tsutsui K, et al. Coagulation-fibrinolysis system and markers of collagen metabolism in lung cancer. Cancer. 1992; 70: 2631-6.

16. Ferrigno D, Buccheri G, Ricca I. Prognostic significance of blood coagulation tests in lung cancer. The European respiratory journal. 2001; 17: 667-73.

17. Nakano K, Sugiyama K, Satoh H, Shiromori S, Sugitate K, Arifuku H, et al. Risk factors for disseminated intravascular coagulation in patients with lung cancer. Thoracic cancer. 2018:9:931-8.
18. Skorek P, Stępień K, Fila M, Hauer J, Kużdżał J. Preoperative thrombocytosis in surgically treated patients with non-small cell lung cancer. Polish archives of internal medicine. 2018; 128: 512-7.

19. Fan S, Zhao G, An G. High pretreatment plasma D-dimer levels are associated with shorter overall survival in patients with small cell lung cancer. The Journal of international medical research. 2019; 47: 215-24.

20. Zeng $\mathrm{Q}$, Xue N, Dai $\mathrm{D}$, Xing $\mathrm{S}, \mathrm{He} \mathrm{X}, \mathrm{Li} \mathrm{S}$, et al. A Nomogram based on Inflammatory factors C-Reactive Protein and Fibrinogen to predict the prognostic value in patients with resected Non-Small Cell Lung Cancer. Journal of Cancer. 2017; 8: 744-53.

21. van den Berg YW, Osanto S, Reitsma PH, Versteeg HH. The relationship between tissue factor and cancer progression: insights from bench and bedside. Blood. 2012; 119: 924-32.

22. Khalil J, Bensaid B, Elkacemi H, Afif M, Bensaid Y, Kebdani T, et al. Venous thromboembolism in cancer patients: an underestimated major health problem. World journal of surgical oncology. 2015; 13: 204.

23. Gong L, Cumpian AM, Caetano MS, Ochoa CE, De la Garza MM, Lapid DI, et al. Promoting effect of neutrophils on lung tumorigenesis is mediated by CXCR2 and neutrophil elastase. Molecular cancer. 2013; 12: 154.

24. Campello E, Henderson MW, Noubouossie DF, Simioni P, Key NS. Contact System Activation and Cancer: New Insights in the Pathophysiology of Cancer-associated Thrombosis. Thrombosis and haemostasis. 2018; 118: 251-65.

25. Zanetto A, Campello E, Spiezia L, Burra P, Simioni P, Russo FP. Cancerassociated Thrombosis in Cirrhotic Patients with Hepatocellular Carcinoma. Cancers (Basel). 2018; 10: 450

26. Falanga A, Santoro A, Labianca R, De Braud F, Gasparini G, D'Alessio A, et al. Hypercoagulation screening as an innovative tool for risk assessment, early diagnosis and prognosis in cancer: the HYPERCAN study. Thrombosis Research. 2016; 140 (Suppl 1):S55-9.

27. Marinho FC, Takagaki TY. Hypercoagulability and lung cancer. J Bras Pneumol. 2008; 34: 312-22.

28. Pedersen LM, Milman N. Prognostic significance of thrombocytosis in patients with primary lung cancer. The European respiratory journal. 1996: 9: 1826-30.

29. Liu HB, Gu XL, Ma XO, Lv TF, Wu Y, Xiao YY, et al. Preoperative platelet count in predicting lymph node metastasis and prognosis in patients with non-small cell lung cancer. Neoplasma. 2013; 60: 203-8.

30. Russo A., Russano M., Franchina T., Migliorino M.R., Aprile G., Mansueto G, Berruti A., Falcone A., Aieta M., Gelibter A., et al. Neutrophil-to-Lymphocyte Ratio (NLR), Platelet-to-Lymphocyte Ratio (PLR), and Outcomes with Nivolumab in Pretreated Non-Small Cell Lung Cancer (NSCLC): A Large Retrospective Multicenter Study. Advances in therapy. 2020; 37: 1145-55.

31. Diem S, Schmid S, Krapf M, Flatz L, Born D, Jochum W, et al. Neutrophil-toLymphocyte ratio (NLR) and Platelet-to-Lymphocyte ratio (PLR) as prognostic markers in patients with non-small cell lung cancer (NSCLC) treated with nivolumab. Lung cancer. 2017; 111: 176-81. 